\title{
Ventajas e Inconvenientes de la Evaluación Formativa, y su Influencia en la Autopercepción de Competencias en alumnado de Formación Inicial del Profesorado en Educación Física Advantages and Issues of Formative Assessment and its Influence on Self-Perception of Competences in students of Pre-service Physical Education Teacher Training \\ *Francisco Javier Gallardo-Fuentes, **Victor Manuel López-Pastor, Bastian Ignacio Carter-Thuillier *Universidad de Los Lagos (Chile), ${ }^{* *}$ Universidad de Valladolid (España), ${ }^{* * * U n i v e r s i d a d ~ C a t o ́ l i c a ~ d e ~ T e m u c o ~(C h i l e) ~}$
}

Resumen. A juicio de múltiples autores la evaluación es una herramienta fundamental para que el profesor logre sus propósitos en el proceso de Enseñanza-Aprendizaje (E-A), ya que le permite constatar los avances del proceso. Pero además, la evaluación permite al maestro establecer un vínculo con el actor fundamental del proceso educativo: «el alumnado». Los objetivos del estudio son: a- analizar la percepción del alumnado sobre las competencias adquiridas y si varia por la aplicación de un sistema de evaluación formativa y compartida en una asignatura de formación del profesorado de educación física (FIPEF) de una universidad pública chilena. b- conocer la valoración del alumnado sobre dicho sistema de evaluación. Para ello se aplicaron cuestionarios antes y después de haber utilizado un sistema de evaluación formativa y compartida $(\mathrm{EFyC})$ en una asignatura de formación inicial del profesorado en educación física de una universidad pública chilena. El estudio se llevó a cabo bajo diseño pretest-postest con la totalidad del alumnado que cursaba la asignatura, en una muestra de 25 alumnos y alumnas (Edad 23 \pm 1.9$)$. Los principales resultados muestran una valoración positiva hacia el sistema de EFyC, con medias muy altas en los ítems que hacen referencia a las «ventajas» del mismo, mientras que la mayoría de los posibles «inconvenientes» tienen valoraciones bajas o muy bajas. Sólo algunas de estas características (asistencia continua, comprensión previa, continuidad del sistema de evaluación, esfuerzo que exige, trabajo que se puede acumular al final y desproporción trabajo/créditos) son apreciadas como posibles inconvenientes por algunos estudiantes. Además, el sistema de EFyC parece influir positivamente en la percepción de mejora en competencias «transversales»; «docentes generales» y «específicas docentes de educación física».

Palabras clave: Evaluación formativa, evaluación compartida, formación inicial del profesorado, auto-percepción de competencias, alumnado de educación superior.

\begin{abstract}
In the opinion of many authors, assessment is a fundamental tool for teachers to achieve their purposes in the TeachingLearning process, since it allows them to verify the progress of the process. In addition, evaluation allows teachers to establish a link with the fundamental actor of the educational process: «The Student». The objectives of the study are: a- to analyze students' perception of the competencies acquired and whether it varies with the application of a formative and shared evaluation system in a physical education teacher training course (FIPEF) in a Chilean public university. b- to gain knowledge on the assessment of student body with such evaluation system. To do that, questionnaires were applied before and after using a system of formative and shared assessment (F\&SA) in one subject of the Physical Education Teacher Education (PETE) program at a Chilean public university. The study was carried out using a pre-post design with all the students enrolled in the course, a sample of 25 individuals (Age $23 \pm 1.9$ ). The main results show a positive appreciation of the F\&SA system, with very high means in the items that refer to the «advantages» of the system, while most of the possible «disadvantages» show low or very low ratings; only some of these characteristics (continuous assistance, prior understanding, continuity of the evaluation system, effort required, work that can be accumulated at the end, and disproportion of work / credits) are experienced as possible disadvantages by some students. In addition, the F\&SA system seems to positively influence the perception of improvement in the competences «Transversal»; «general teachers» and «specific physical education teachers».
\end{abstract}

Keywords: Formative Assessment, Co-Assessment, Pre-service Teacher Education, skills self-perception, Student‘s Higher Education.

\section{Introducción}

Las competencias suelen relacionarse con las habilidades y destrezas necesarias para desempeñarse adecuadamente en distintos contextos. Numerosas profesiones las adoptan en sus estructuras formativas, diversificando su finalidad y no limitándose a la ejecución de tareas, sino que las competencias profesionales también se vinculan a atributos relacionados con el saber, saber hacer, saber estar y saber ser (Proyecto Tunning, 2003). Pueden encontrarse diferentes definiciones para el concepto de «competencias». Para Yániz \& Villardón (2006, p.23) son «el conjunto de conocimientos, habilidades y actitudes necesarios para desempeñar una ocupación». Por su parte, Zabalza (2003, p.70) las define como el «conjunto de conocimientos y habilidades que los sujetos necesitamos para desarrollar algún tipo de

Fecha recepción: 11-08-19. Fecha de aceptación: 24-02-20 Francisco Gallardo Fuentes

fgallardo@ulagos.cl actividad». A pesar de emerger del mundo laboral, algunos autores coinciden en que las competencias tienen cabida en una visión educativa (Burnier, 2001; Díaz-Barriga, 2006; Perrenoud, 2002; Toboìn, Rial, Carretero \& García, 2006).

En los contextos formativos las competencias se transforman en modelo, y como nuevo paradigma educativo es idóneo y efectivo para el proceso de enseñanza-aprendizaje (E-A) actual (De Miguel, 2005); además, en el contexto universitario centran su acción en aprendizajes orientados a la práctica profesional con un marcado protagonismo del alumnado en su propio proceso formativo (López, Benedito \& León, 2016). En el cambio hacía este nuevo paradigma educativo, al que muchas instituciones universitarias están transitando, surge una condición que, sin duda, marca la diferencia, ya que plantea otro enfoque del trabajo docente en el aula, en que la labor del profesorado se centra en los procesos de aprendizaje del alumnado y no en la docencia del profesorado (Tejada, 2009). En un modelo por competencias el profesorado no está para imponer su criterio, sino que debe utilizar como herramienta el diálogo con el alumnado y, 
de esta manera, «conocer sus intereses, ver qué les motiva, comprobar cuáles son sus preocupaciones, para luego integrar todo esto en la vida del aula» (Barba, 2009, p.43), procurando avanzar hacia una evaluación autentica (CalatayudSalom, 2019).

Los sistemas competenciales se implementan como obligatorios en las universidades del Espacio Europeo de Educación Superior (EEES) a partir de la declaración de Bolonia (Declaración Bolonia, 1999). Por su parte, en el Espacio Chileno de Educación Superior (ECES), hay antecedentes de la implementación de «currícula» por competencia a partir del año 2005 (Hawes \& Corvalán, 2005).

En estos sistemas formativos, la evaluación no está únicamente centrada en conocer el nivel de los alumnos en torno a determinadas competencias, sino que se propone una mirada educativa centrada en el conocimiento, con el fin de favorecer la construcción gradual de los aprendizajes desde una mirada constructivista (Lasnier, 2000), por lo que propone ver «la evaluación de competencias como un planteamiento videográfico y no fotográfico. En efecto, no se trata tanto de emitir un juicio al final del trayecto, sino de seguir la progresión del desarrollo de competencias» (Fernández, 2010, p.16). Siendo necesaria una constante actualización docente, que le permita al profesorado comprender las características del alumnado (Fernández-Rivas \& Espada-Mateos, 2017).

Ante este nuevo paradigma surgen preguntas como: ¿qué evaluamos? y ¿cómo evaluamos? Dar respuesta no es fácil, especialmente si tomamos en cuenta que según Boud et al. (2010), la evaluación es la que condiciona qué y cómo se aprende (Biggs, 2005); esto puede comprobare en contextos donde la tendencia es usar casi exclusivamente la heteroevaluación, que se presenta como una práctica que obstaculiza el aprendizaje (Canales-Núñez, Aravena, Carcamo-Oyarzún, Lorca \& Martínez-Salazar, 2018).

En una búsqueda de experiencias que pudiesen dar respuesta adecuada a las preguntas antes planteadas, nos hemos encontrado con la existencia de una red que surge en el año 2005 por profesores universitarios de distintos países de habla hispana, denominada «Red de evaluación formativa y compartida en educación superior (REFYCE)», que cuenta con trabajos sistemáticos y documentados en este ámbito (Gallardo-Fuentes \& Carter-Thuillier, 2016; López-Pastor, 2009; Hamodi, López \& López-Pastor, 2014; López-Pastor, Pérez, Barba \& Lorente, 2016).

En este sentido, la REFYCE plantea que la evaluación formativa y compartida (EFyC), tiene el potencial de insertarse en el proceso de E-A y estar orientada a que el alumno aprenda más y que el profesorado perfeccione su práctica docente (López-Pastor, 2009). Pueden encontrarse otros trabajos en esta línea. Por ejemplo:

Rodríguez, Del Valle \& De la Vega (2018, p.387) considera que «El alumno es protagonista de su propio aprendizaje y el profesor de su propia enseñanza. Pero además, aunque entre ambos exista un nivel de partida diferente, el profesor cuando enseña, aprende y el alumno cuando aprende es capaz de enseñar, construyendo una relación dialéctica entre ambos influyendo cada uno en el crecimiento personal del otro y por consiguiente en el de la sociedad».

Según Martínez, Santos-Pastor \& Castejón-Oliva (2017, p.80), para esto es necesaria una comunicación activa en el proceso de E-A. En este mismo sentido, Barrientos, LópezPastor \& Pérez-Brunicardi (2019) consideran que la falta de entendimiento entre el profesorado y el alumnado no es solo una cuestión de pareceres, sino una falta de compromiso de ambas partes para que se consigan los aprendizajes y competencias necesarias en la formación inicial del profesorado. Volviendo al planteamiento inicial de la red, en López-Pastor, (2009) se explica claramente que la evaluación formativa mejora la implicación del alumnado en su propio aprendizaje, al ser un «proceso de constatación, valoración y toma de decisiones cuya finalidad es optimizar el proceso de enseñanzaaprendizaje que tiene lugar, desde una perspectiva humanizadora y no como mero fin calificador» (p.35).

Los objetivos de este estudio son:

a) Conocer la valoración del alumnado que realiza el alumnado sobre el sistema de EFyC utilizado, en una asignatura de FIPEF en una universidad pública chilena.

b) Analizar la percepción del alumnado sobre tres tipos de competencias adquiridas (transversales, docentes genéricas y docentes específicas de educación física); tanto antes como después de haber aplicado un sistema de EFyC a lo largo de una asignatura en la FIPEF de una universidad pública chilena.

\section{Material y Métodos}

El estudio tiene un diseño pretest-postest, estableciendo el grado de relación que existe entre la percepción de competencias adquirida antes y después de haber implementado un sistema de EFyC en una asignatura universitaria de FIPEF en una universidad chilena.

\section{Población y muestra}

La muestra está formada por 25 alumnos y alumnas, de (edad 23 \pm 1.9 ) años, que son la totalidad del alumnado que cursó la asignatura «Gasto Energético y Antropometría», correspondiente al cuarto semestre de FIPEF en una universidad pública chilena, con una duración de 18 semanas (agosto-diciembre).

\section{Sistema de evaluación utilizado}

Se decidió aplicar un sistema de EFyC, dadas las múltiples experiencias que lo consignan como positivo para el proceso de E-A(Castejón, López-Pastor, Julián \& Zaragoza, 2011; Gallardo-Fuentes \& Carter-Thuillier, 2016; López-Pastor, 2008; López-Pastor, Pérez, Barba \& Lorente, 2016; Martín, 2007).

El sistema de evaluación utilizado está centrado en los procesos de aprendizaje del alumnado y sirve para reforzar y orientar el esfuerzo del alumnado en los aprendizajes a realizar. En la tabla 1 se explican las relaciones entre «actividades de aprendizaje», «sistema de evaluación formativa durante el proceso de aprendizaje», «técnicas e instrumentos de evaluación» y «peso en la calificación final de cada actividad de aprendizaje». Dado que la experiencia se ha llevado a cabo en Chile, el sistema de calificación final respondía a una escala $0-7$, en donde el aprobado se ubicaba en la nota 4.

Como se puede ver en la tabla 1, es fundamental que todas las actividades de aprendizaje estén asociadas a pro- 
Tabla

Relaciones entre Actividades de Aprendizaje, Proceso de Evaluación Formativa, Instrumentos Utilizados y Peso en la Calificación Final.

\begin{tabular}{|c|c|c|c|c|}
\hline $\mathbf{N}^{\circ}$ & $\begin{array}{l}\text { Actividades de } \\
\text { aprendizaje }\end{array}$ & $\begin{array}{l}\text { Actividades formativas y de } \\
\text { retroalimentación }\end{array}$ & $\begin{array}{l}\text { Instrumento de } \\
\text { evaluación }\end{array}$ & $\begin{array}{l}\text { Peso en la } \\
\text { calificación } \\
\text { final }\end{array}$ \\
\hline 3 & $\begin{array}{l}\text { Clases Teóricas } \\
\text { dirigidas por el Profesor, } \\
\text { con debate y } \\
\text { participativa }\end{array}$ & $\begin{array}{l}\text { "Evaluaciones de Proceso". } \\
\text { Retroalimentación del } \\
\text { profesor en pruebas escritas, } \\
\text { interrogantes en clase, etc. }\end{array}$ & $\begin{array}{l}\text { Escala de puntos } \\
\text { al } 60 \% \\
\text { exigencia }\end{array}$ & $40 \%$ \\
\hline 1 & $\begin{array}{l}\text { Clases prácticas } \\
\text { dirigidas por alumnado, } \\
\text { con interacción del } \\
\text { profesor }\end{array}$ & $\begin{array}{l}\text { "Evaluación integradora de } \\
\text { proceso". Supervisión y } \\
\text { feedback verbal por parte del } \\
\text { profesor. }\end{array}$ & $\begin{array}{l}\text { Plantillas de } \\
\text { planificación de } \\
\text { entrenamiento }\end{array}$ & $20 \%$ \\
\hline 1 & $\begin{array}{l}\text { Diseño de Proyecto con } \\
\text { exposición ante pares }\end{array}$ & $\begin{array}{l}\text { "Evaluación orientada al } \\
\text { desempeño profesional". Se } \\
\text { entregó retroalimentación en } \\
\text { horarios de atención de } \\
\text { alumnado. }\end{array}$ & Rubrica & $25 \%$ \\
\hline 1 & $\begin{array}{l}\text { Proceso reflexivo de } \\
\text { cierre, con interacción } \\
\text { del profesor }\end{array}$ & $\begin{array}{l}\text { "Autoevaluación". Terminada } \\
\text { la asignatura y de manera } \\
\text { dialogada con el profesor, el } \\
\text { alumnado se autoevalúa }\end{array}$ & $\begin{array}{l}\text { Pauta de } \\
\text { autoevaluación }\end{array}$ & $15 \%$ \\
\hline
\end{tabular}

cesos de EFyC, que faciliten un constante feedback entre el profesor y el alumnado, así como la oportunidad de corregir y mejorar los documentos o actividades. También considera lo planteado por Cabrera y Mayordomo, ya que el alumnado «valora que se les proporcionen directrices de lo que se espera de ellos» (2016, p.17).

\section{Instrumentos de obtención de datos}

Para conocer la valoración de parte del alumnado hacia el sistema de EFyC empleado, y determinar sus efectos en la percepción de adquisición de competencias, se les aplicó durante el semestre dos instrumentos de obtención de datos:

1. El «Cuestionario de evaluación sobre la experiencia de buena práctica». Cuestionario con escala tipo likert de cinco grados (de 0 a 4 , en donde $0=$ Nada; $1=$ Poco; $2=$ Algo; $3=$ Bastante; $4=$ Mucho), en respuesta a las afirmaciones contenidas en una serie de ítems (ver tablas de resultados II y III). Dicho cuestionario ha sido previamente validado (Castejón-Oliva, Pastor \& Palacios, 2015), presentando un coeficiente de .84 Alpha de Cronbach para toda la escala. En el presente estudio el análisis se centró en los ítems y variables que dan respuesta a los siguientes factores:

a) Ventajas del sistema de Evaluación ( 18 ítems; $\mathrm{n}^{\circ} 11 . \mathrm{x}$ ).

b) Posibles desventajas del sistema de evaluación (13 ítems; $\left.\mathrm{n}^{0} 12 . \mathrm{x}\right)$

2. Una «Escala para la autopercepción de competencia de los estudiantes». Escala tipo likert de cuatro grados (de 1 a 4 , en donde $1=$ Nada; $2=$ Poco; $3=$ Bastante y $4=\mathrm{Mu}$ cho), para responder a los ítems (ver tablas de resultados IV, $\mathrm{V}$ y VI) contenidos en los tres bloques que componían el instrumento. Esta escala ha sido previamente validada (Salcines-Talledo, González-Fernández, Ramírez-García \& Martínez-Mínguez, 2018).

\section{Análisis estadísticos y de datos}

Para el análisis de datos se utilizó estadística descriptiva, en cada variable del «Cuestionario sobre la experiencia de buena práctica», por medio de la media aritmética $(M)$ y la desviación típica ( $d t$.). Además de análisis de diferencia de medias entre las percepciones del alumnado en la «Escala para la autopercepción de competencia de los estudiantes» entre el pretest y el post-test aplicado al alumnado; para esto se utilizó estadística inferencial, con la prueba de $U$ de Mann-Whitney $(U)$, en un nivel de significación de $p \mathrm{~d} \gg .05$. La elección de esta prueba responde a los resultados ade- cuados que ha demostrado al ser aplicada para analizar escalas de tipo Likert, donde la prueba $U$ de Mann-Whitney $(U)$ posee una potencia equivalente a la prueba t-Student's, independiente del tamaño de las muestras (De Winter \& Dodou, 2010).

\section{Resultados}

\section{Evaluación del sistema de EFyC de la asignatura}

En la tabla 2 se presenta el grado de acuerdo a las afirmaciones referentes al primer factor «Ventajas del sistema de Evaluación».

\begin{tabular}{|c|c|c|}
\hline Preguntas & $M$ & dt. \\
\hline \multicolumn{3}{|l|}{$\begin{array}{l}\text { 11. Señala si estás de acuerdo con las siguientes afirmaciones relativas al sistema } \\
\text { de evaluación de la asignatura: }\end{array}$} \\
\hline 11.1. Ofrece alternativas a todos los estudiantes & 3.52 & .51 \\
\hline 11.2. Hay un contrato previo, negociado y consensuado del sistema de evaluación & 3.64 & 1.04 \\
\hline 11.3. Esta centrado en el proceso, importancia del trabajo diario & 3.36 & .49 \\
\hline 11.4. El estudiante realiza un aprendizaje activo & 3.40 & .58 \\
\hline 11.5. Se plantea el trabajo en equipo de forma colaborativa & 3.20 & .58 \\
\hline 11.6. El alumno está más motivado, el proceso de aprendizaje es más motivador & 3.00 & .71 \\
\hline 11.7. La calificación es más justa & 3.28 & .61 \\
\hline 11.8. Mejora la tutela académica (seguimiento y ayuda al alumno) & 2.96 & .61 \\
\hline 11.9. Permite aprendizajes funcionales & 3.24 & .60 \\
\hline 11.10. Permite aprendizajes significativos & 3.32 & .63 \\
\hline 11.11. Se aprende mucho mas & 3.40 & .58 \\
\hline 11.12. Mejora la calidad de los trabajos exigidos & 3.32 & .63 \\
\hline 11.13. Hay interrelación entre teoría y practica & 3.44 & .71 \\
\hline 11.14. Evalúa todos los aspectos posibles (saber, saber hacer, saber & 3.48 & .59 \\
\hline 11.15. Hay retroalimentación en documentos y actividades & 3.28 & .79 \\
\hline 11.16. Hay posibilidad de corregir errores en documentos y actividades & 2.88 & .88 \\
\hline 11.17. Se da un seguimiento más individualizado & 2.52 & .77 \\
\hline 11.18. Requiere más responsabilidad & 3.20 & .71 \\
\hline
\end{tabular}

Los resultados muestran (ver tabla 2), que las afirmaciones contenidas en «ventajas del sistema de evaluación» manifiestan un grado de acuerdo muy alto, entre «bastante» $\mathrm{y}$ «mucho».

Por tanto, el alumnado valora muy positivamente las ventajas del sistema de evaluación utilizado. Destacan especialmente las ventajas que hacen referencia a que el sistema ofrece alternativas a todos los estudiantes, que ha sido consensuado con los estudiantes y que evalúa todos los aspectos posibles del saber, con medias muy altas.

En la tabla 3 presentaremos el grado de acuerdo para las afirmaciones del factor «Posibles inconvenientes del sistema de evaluación».

Tabla 3

Medias y dt. Factor "Posibles Inconvenientes del Sistema de Evaluación"

\begin{tabular}{lll}
\hline Preguntas & $\boldsymbol{M}$ & $\boldsymbol{d t .}$ \\
\hline $\begin{array}{l}\text { 12. Señala si estás de acuerdo con las siguientes afirmaciones relativas } \\
\text { sistema de evaluación de la asignatura: }\end{array}$ & \\
12.1. Exige una asistencia obligatoria y activa & 3.40 & .58 \\
12.2. Tiene una dinámica de trabajo poco conocida, falta de hábito & 2.52 & .92 \\
12.3. Exige continuidad & 3.28 & .74 \\
12.4. Hay que comprenderlo previamente & 2.96 & .61 \\
12.5. Exige un mayor esfuerzo & 3.04 & .79 \\
12.6. Existe dificultad para trabajar en grupo & 1.96 & 1.14 \\
12.7. Se puede acumular mucho trabajo al final & 2.16 & 1.07 \\
12.8. Existe una desproporción trabajo/créditos & 2.20 & 1.26 \\
12.9. El proceso de calificación es más complejo y, a veces, poco claro & 1.68 & 1.11 \\
12.10. Genera inseguridad e incertidumbre, dudas sobre qué hay que realizar & 1.84 & 1.07 \\
12.11. Es injusto frente a otros procesos de evaluación & 1.44 & 1.26 \\
12.12. Las correcciones han sido poco claras & 1.36 & 1.11 \\
12.13. La valoración del trabajo es subjetiva & 1.96 & 1.17 \\
\hline Fuente: elaboración propia & &
\end{tabular}

En la tabla 3 se puede apreciar dos bloques de ítems. Por un lado, valoraciones medias y altas para las afirmaciones que hacen referencia a la asistencia obligatoria, continuidad, comprensión previa, exigencia de mayor esfuerzo, acumulación de trabajo y desproporción trabajo/créditos del sistema de EFyC. En estos ítems la valoración del alumnado se encuentra en un nivel medio/alto (2.52-3.40 sobre 4). Por otro lado, un bloque de afirmaciones correspondientes a posi- 
bles inconvenientes que se dan entre nada y poco (1.36-1.96 sobre 4), en donde se encuentran las afirmaciones: «Existe dificultad para trabajar en grupo»; «El proceso de calificación es más complejo y, a veces, poco claro»; «Genera inseguridad e incertidumbre»; «Es injusto frente a otros procesos de evaluación»; «Las correcciones han sido poco claras» $\mathrm{y}$ «La valoración del trabajo es subjetiva».

\section{Autopercepción de competencias del alumnado}

Hemos organizado los resultados sobre la autopercepción de competencia por parte del alumnado en función de los tres bloques de competencias analizados.

En la tabla 4 pueden observarse las percepciones del alumnado para el bloque I, correspondiente a las «competencias transversales».

\begin{tabular}{|c|c|c|c|c|c|c|}
\hline Ítems & $\begin{array}{c}M \\
\text { pre-t }\end{array}$ & $d t$. & $\begin{array}{c}M \\
\text { post-t }\end{array}$ & $d t$ & $U$ & Sig. \\
\hline Bl.1. Analizar y sintetizar & 2.74 & .62 & 3.00 & .60 & 207.5 & .149 \\
\hline B1.2. Organizar y planificar & 2.61 & .50 & 3.09 & .67 & 165 & $.013^{*}$ \\
\hline B1.3. Comunicarse de forma oral y escrita en lengua nativa & 2.74 & .69 & 3.13 & .69 & 186.5 & .062 \\
\hline B.4. Comunicarse gestual y corporalmente & 3.09 & .60 & 2.91 & .51 & 225.5 & .290 \\
\hline B1.5. Conocer una lengua extranjera & 2.26 & .62 & 1.78 & .67 & 170.5 & $.018^{*}$ \\
\hline $\begin{array}{l}\text { B1.6. Utilizar las Tecnologías de la Información y la } \\
\text { comunicación en el ámbito de estudio }\end{array}$ & 3.00 & .67 & 2.96 & .71 & 255.5 & .827 \\
\hline B1.7. Trabajar en equipo & 3.52 & .59 & 3.57 & .59 & 253.5 & .780 \\
\hline $\begin{array}{l}\text { B1.8. Desarrollar habilidades en las relaciones interpersonales } \\
\text { (empatia, asertividad, respeto y escucha) }\end{array}$ & 3.61 & .50 & 3.48 & .59 & 237 & .485 \\
\hline $\begin{array}{l}\text { B1.9. Desarrollar habilidades intrapersonales (autoestima, } \\
\text { motivación y confianza en uno mismo) }\end{array}$ & 3.48 & .51 & 3.43 & .59 & 258.5 & .880 \\
\hline B1.10. Razonar de forma crítica y reflexiva & 3.17 & .49 & 3.17 & .58 & 262.5 & .957 \\
\hline B.11. Desarrollar un compromiso ético & 3.35 & .57 & 3.17 & .78 & 236.5 & .499 \\
\hline B1.12. Desarrollar un aprendizaje autónomo & 3.00 & .67 & 3.30 & & 201.5 & .116 \\
\hline B1.13. Adaptarse a situaciones nuevas & 3.09 & .60 & 3.30 & .47 & 217.5 & .209 \\
\hline B1.14. Desarrollar la creatividad & 3.22 & .60 & 3.30 & .56 & 246 & 637 \\
\hline
\end{tabular}

Los resultados muestran que la percepción del alumnado se encuentra en un nivel alto (entre bastante y mucho) al inicio y termino de la asignatura, salvo en un ítem («Conocer una lengua extranjera»). También se puede apreciar que, según la valoración del alumnado, la experiencia de EFyC aporta a la adquisición de competencias transversales dada la valoración por sobre el pretest en seis ítems («Organizar y planificar»; «Comunicarse de forma oral y escrita en lengua nativa»; «Trabajar en equipo»; «Desarrollar un aprendizaje autónomo»; «Adaptarse a situaciones nuevas» y «Desarrollar la creatividad»). Por otro lado, la experiencia de EFyC no aporto en la adquisición de las competencias transversales contenidas en los ítems: "Comunicarse gestual y corporalmente»; «Conocer una lengua extranjera»; «Utilizar las Tecnologías de la Información y la comunicación en el ámbito de estudio»; «Desarrollar habilidades en las relaciones interpersonales (empatía, asertividad, respeto y escucha)»; «Desarrollar habilidades intrapersonales (autoestima, motivación y confianza en uno mismo)» y «Desarrollar un compromiso ético», donde el valor del postest fue más bajo. Finalmente se puede apreciar que solo existen dos ítems presentan diferencias estadísticamente significativas, indicándonos que el sistema de evaluación fue un aporte en «Organizar y planificar», sin embargo, no aportó a «Conocer una lengua extranjera».

En la Tabla 5 se presentan los resultados a los ítems correspondientes a las «competencias docentes generales».

La mayoría de los ítems recibieron valoraciones medias y altas (entre 2.22 y 2.09 en una escala 1-4, tanto antes como después de aplicar el sistema de EFyC. También se puede

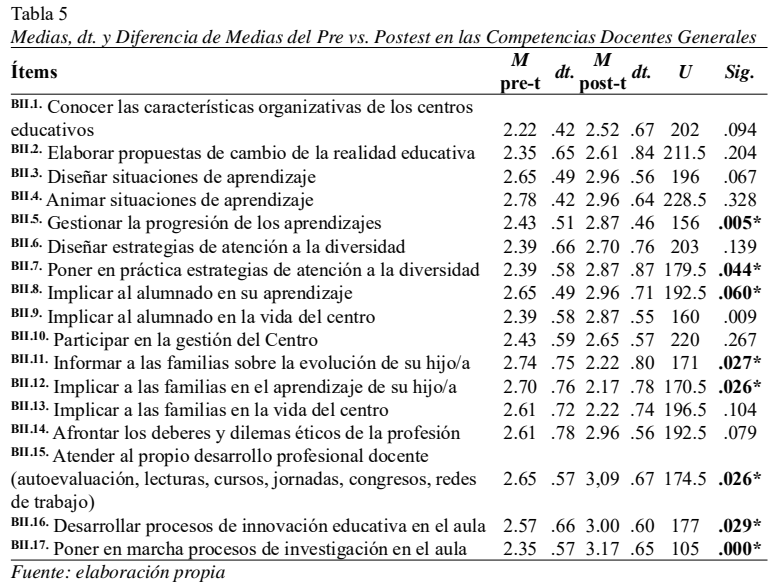

apreciar que en siete ítems aparecen diferencias significativas entre pretest y el postest. Estos ítems se dividen en dos grupos. Los del primer grupo dejan ver un aporte significativo de la experiencia de EFyC en la adquisición de competencias docentes; por ejemplo, acá encontramos que a juicio del alumnado el sistema de evaluación utilizado les aportó, y lo dejan ver con valoraciones significativamente mas altas en el postest en seis ítems: «Gestionar la progresión de los aprendizajes»; «Poner en práctica estrategias de atención a la diversidad»; «Implicar al alumnado en su aprendizaje»; «Atender al propio desarrollo profesional docente (autoevaluación, lecturas, cursos, jornadas, congresos, redes de trabajo)»; «Desarrollar procesos de innovación educativa en el aula»y «Poner en marcha procesos de investigación en el aula».

El segundo grupo lo componen dos ítems («Informar a las familias sobre la evolución de su hijo/a» y «Implicar a las familias en el aprendizaje de su hijo/a») que obtienen valoraciones estadísticamente significativamente más bajas en el postest.

\section{Tabla 6}

Medias, dt. y Diferencia de Medias del Pre vs. Postest en las Competencias Específicas Docentes de Educación Física

\begin{tabular}{lcccccc}
\hline Ítems & $\begin{array}{c}M \\
\text { pre-t }\end{array}$ & $d t$. & $\begin{array}{c}M \\
\text { post-t }\end{array}$ & $d t$. & $U$ & Sig.
\end{tabular}

BIII.1. Diseñar, aplicar y analizar intervencion

didácticas en el área de Educación Física

III.2. Diseñar, desarrollar y evaluar los procesos de

$\begin{array}{lllllll}\text { enseñanza-aprendizaje relativos a la actividad física y el } & 2.65 & .57 & 2.87 & .55 & 213.5 & .187\end{array}$ deporte con atención a las Necesidades Específicas de

Apoyo Educativo

BIII.3. Conocer y promover las diferentes

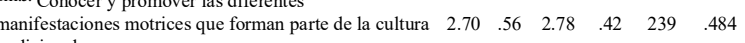

tradicional

BIII.4. Conocer y aplicar los fundamentos biológicos,

fisiológicos, de maduración evolutiva y el desarrollo

psicomotor

BIII.5. Diseñar, modificar $\mathrm{y} / \mathrm{o}$ adaptar al contexto

educativo situaciones motrices orientadas al desarrollo

y perfeccionamiento de las habilidades motrices

BIII.6. Conocer los elementos y fundamentos de la

expresión corporal y la comunicación no verbal y su $\quad \begin{array}{lllllll}2.48 & .51 & 3.04 & .64 & 145 & \mathbf{. 0 0 3} \text { * }\end{array}$

valor formativo y cultural

BIII.7. Saber utilizar el juego como recurso didáctico y $\quad \begin{array}{llllllll}3.17 & .58 & 2.65 & .98 & 183 & \mathbf{. 0 5 3}\end{array}$

como contenido de enseñanza

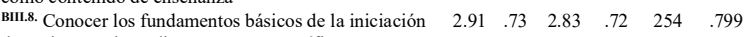
deportiva escolar y diseñar tareas específicas para

utilizarlos en el ámbito de la enseñanza

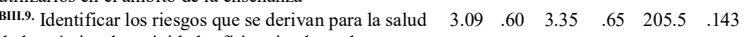

de la práctica de actividades fisicas inadecuadas

BIII.10. Diseñar un plan de hábitos de vida saludable

(higiene y alimentación) y de práctica de actividad

física regular

BIII.11. Saber aplicar los fundamentos (técnicas) de las

actividades fisicas en el medio natural

BIII.12. Saber utilizar diferentes instrumentos de

evaluación en el área de Educación Física

ens. Tener capacidad de reflexión sobre el proceso d

dentro de las clases de Educación Física

dentro de las clases de Educación Física
BIII.14. Analizar y comunicar, de manera crítica y

fundamentada, el valor de la actividad fisica y el

deporte y sus posibilidades de contribuir al desarrollo y

bienestar de las personas

buente: elaboración propia

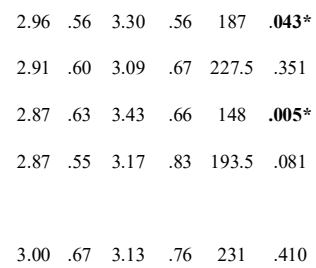

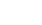


En la Tabla 6 pueden verse los resultados de las percepciones a las «competencias específicas docentes de educación física» y las diferencias de medias entre el pretest y el postest.

La mayoría de los ítems recibieron valoraciones altas (entre 2.48 y 3.43 en una escala $1-4$ ), tanto previo como posterior al sistema de EFyC empleado. Al igual que en el bloque anterior, las diferencias significativas se dividen en dos grupos: (1) las significativamente más altas en el postest que corresponde a las afirmaciones «Conocer y aplicar los fundamentos biológicos, fisiológicos, de maduración evolutiva y el desarrollo psicomotor»; «Conocer los elementos y fundamentos de la expresión corporal y la comunicación no verbal y su valor formativo y cultural»; «Diseñar un plan de hábitos de vida saludable (higiene y alimentación) y de práctica de actividad física regular» $\mathrm{y}$ «Saber utilizar diferentes instrumentos de evaluación en el área de Educación Física», (2) las significativamente más altas en el pretest, que hace referencia a: «Saber utilizar el juego como recurso didáctico y como contenido de enseñanza».

\section{Discusión}

Estos resultados coinciden con estudios previos sobre la temática, realizados en su mayoría en la universidad española (Atienza, Valencia-Peris, Marts-García, López-Pastor \& Devís-Devís, 2016; Hortigüela, Abella \& Pérez-Puello, 2015; Hortigüela, Pérez-Pueyo \& Abella, 2015; López-Pastor \& Pérez-Pueyo, 2017). Por tanto, una aportación del artículo es que parece reforzar la idea de que los sistemas de EFyC también obtienen buenos resultados en la universidad chilena, en la que hasta el momento menos estudios similares sobre la temática (Gallardo-Fuentes \& Carter-Thuillier, 2016). Por tanto, parece existir una adecuada transferencia en la aplicación de los sistemas de EFyC entre la FIP en la universidad española y la universidad chilena.

Las valoraciones más altas hacen referencia a las siguientes características: «Ofrece alternativas a todos los estudiantes» y «Hay un contrato previo, negociado y consensuado del sistema de evaluación». Estos resultados concuerdan con los encontrados por otros estudios sobre la temática, en los que se defiende que la EFyC ayuda a lograr una mayor implicación del alumnado en su propio proceso de aprendizaje (Angelini, 2016; Atienza et al., 2016; Hortigüela et al., 2015; Vallés, Ureña \& Ruiz, 2011; Zaragoza, Luis-Pascual \& Manrique, 2009; Barrientos, López-Pastor \& PérezBrunicardi, 2019); además de otras investigaciones, donde se enfatiza la importancia de hacer participe al alumnado en los procesos de evaluación (Delgado, Ausin, Hortigüela \& Abella, 2016; Rodríguez, Del Valle \& De la Vega, 2018; Calatayud-Salom, 2019).

Con respecto a los «Posibles Inconvenientes del Sistema de Evaluación», los resultados muestran dos grupos claramente diferentes. Por un lado, existe un bloque de ítems que alcanza valores medios/altos (2.52-3.40 sobre 4); son los que hacen referencia a temas como: asistencia continua, comprensión previa, continuidad del sistema de evaluación, esfuerzo que exige, trabajo que se puede acumular al final y desproporción trabajo/créditos. Más que inconvenientes, se podría considerar que son características que debe tener todo sistema de EFyC que se base en el aprendizaje y evaluación continua (López-Pastor, 2009). Ahora bien, pueden encontrarse diferentes estudios que muestran este tipo de resistencias, como el de Fraile \& Cornejo (2013), en el que más del 90\% de los participantes indicó que el sistema de EFyC implementado presentaba como inconveniente «la asistencia obligatoria», «la participación activa» y «el mayor esfuerzo que exige». Por otra parte, en referencia a la «comprensión previa», el estudio de Zaragoza, Luis-Pascual \& Manrique (2009) muestra que el $87 \%$ del alumnado manifiesta falta de costumbre ante este tipo de evaluación, sintiéndose perdidos e incómodos; además, en el estudio de RomeroMartín, Castejón-Oliva \& López-Pastor (2015) el alumnado indicaba como inconveniente el trabajo acumulado al final del proceso. También se encuentran percepciones de mayor esfuerzo y exigencia tras la aplicación de sistemas de EFyC en otros estudios, como en el de Hortigüela, Pérez-Pueyo \& Abella (2015), que en una muestra de 3.618 estudiantes el $50 \%$ encontró una desproporción trabajo/créditos. En cambio, algunos estudios que revisan numerosas experiencias de EFyC en la universidad, con muestras grandes, parecen indicar que los datos empíricos sobre la carga de trabajo que supone para el alumnado un sistema de EFyC suelen estar dentro de los márgenes que establecen los créditos oficiales ECTS (European Credit Transfer and Accumulation System) para el trabajo no presencial que hay que realizar en cada asignatura (López-Pastor, Pintor, Muros \& Webb, 2013; Romero-Martín, Fraile-Aranda, López-Pastor \& Castejón-Oliva, 2014; Atienza et al., 2016; Julián, Zaragoza, CastejónOliva \& López-Pastor, 2010), otra cosa es que el alumnado acostumbrado sólo a asistir a clase durante tres meses y limitarse a estudiar mucho en las últimas semanas anteriores al examen lo viva como inconvenientes; lo cual puede generarnos la duda de si esas percepciones de mayor carga de trabajo no se deben más bien a una comparativa con los sistemas tradicionales (en los que la carga de trabajo se acumula solo en las semanas previas al examen). En cierta relación con esto, otros estudios indican que, aunque el profesorado considera que evalúa de manera adecuada, manifiesta que podría hacerlo mucho mejor si contara con más tiempo para ello (Martínez, Santos-Pastor \& Castejón-Oliva, 2017).

Por otro lado, existe otro bloque de ítems que alcanza valores bajos (1.36-1.96 sobre 4), lo que indica que el alumnado considera que ocurre entre nada y poco. Son los que hacen referencia a temas como: «la dificultad para trabajar en grupo», «la complejidad y falta de claridad del sistema de evaluación», «la inseguridad e incertidumbre sobre lo que hay que realizar», «la injusticia frente a otros tipos de evaluación», «la poca claridad en las correcciones y la valoración subjetiva de los trabajos». Se pueden encontrar datos similares como el estudio de Hortigüela et al., (2015) donde el $45.2 \%$ del alumnado de cursos iniciales considera que existe inseguridad e incertidumbre al enfrentarse a sistemas de EFyC, mientras que solo el $2.2 \%$ del alumnado de cursos finales cree en la existencia de esta inseguridad e incertidumbre; eso sí, ambos grupos valoran el sistema de EFyC como altamente clarificador en sus correcciones. Por otra parte, también se encuentran estudios que contrastan con los resultados del presente estudio (Gallardo-Fuentes \& Carter-Thuillier, 2016; Zaragoza et al., 2009), presentando altas valoraciones a este 
tipo de ítems; muchas veces estas responden a resistencias del alumnado que está acostumbrado a un modelo tradicional y que lo prefiere, dado que supone una menor carga de trabajo y una mayor libertad a lo largo de los tres primeros meses de cada cuatrimestre (López-Pastor, 2009). En todo caso, estas percepciones no se ven como verdaderos inconvenientes, sino como elementos propios de un sistema de EFyC, y que en diferentes estudios el alumnado asume que la elevada carga de trabajo y esfuerzo se ve recompensada con un mayor aprendizaje y rendimiento académico (LópezPastor \& Pérez-Pueyo, 2017; Romero-Martín et al., 2014). También hay estudios que plantean que la EFyC podría ayudar en la implicación del alumnado en proceso E-A (Hortigüela, Pérez-Pueyo \& López-Pastor, 2015), y esta implicación puede ser mayor cuando el alumnado participa más veces de sistemas de EFyC a lo largo de la carrera (LópezPastor, 2009). Además, hay experiencias que muestran mejoras en la independencia y conciencia del alumnado sobre su propio aprendizaje (Senye-Mir, Arumí-Prat, Pla-Campas \& Ramírez, 2016; Zaragoza et al., 2009), encontrándose incluso casos en que el alumnado que se ve involucrado en procesos de EFyC con criterios claros desde el comienzo, identifican al sistema de evaluación como generador de aprendizaje, con una alta valoración, independiente de la calificación que este sistema les entregue (Espinoza, 2013).

En referencia a la autopercepción de competencias por parte del alumnado, se presentaron diferencias estadísticamente significativas entre las medias del pre y postest. Las diferencias más elevadas se encuentran tras la implementación del sistema de EFyC en las Competencias transversales, especialmente en la denominada capacidad de «Organizar y planificar», en esta misma línea se coincide con el estudio de Silva \& López-Pastor (2015), donde el alumnado indica que la EFyC ayuda a la capacidad de organización, así como con la experiencia desarrollada en la Universidad Autónoma de Barcelona, en donde tras la aplicación de proyectos de aprendizaje tutorados (PAT) el alumnado manifestó una percepción alta para la competencia de «organización» tras la experiencia (Martínez-Mínguez, 2016).

En lo relativo a las Competencias docentes generales, hay diferencias estadísticamente significativas más elevadas para el postest en las competencias: «Gestionar la progresión de los aprendizajes», «Poner en práctica estrategias de atención a la diversidad», «Implicar al alumnado en su aprendizaje», «Atender al propio desarrollo profesional docente (autoevaluación, lecturas, cursos, jornadas, congresos, redes de trabajo)», «Desarrollar procesos de innovación educativa en el aula» y «Poner en marcha procesos de investigación en el aula». Pueden encontrarse resultados similares en otros estudios sobre competencias de este tipo tras la aplicación de sistemas de EFyC (López-Pastor, 2011; Santos-Pastor, Martínez-Muñoz \& López-Pastor, 2009; Ureña-Ortín \& Ruiz-Lara, 2012). Además, los resultados del presente estudio son coherentes con la finalidad de este tipo de sistemas de evaluación, ya que López-Pastor (2009) afirma que los sistemas de EFyC ayudan a perfeccionar la práctica docente y, por ende, las competencias comprometidas en la misma.

Por último, en las Competencias específicas docentes de educación física, las diferencias estadísticamente significa- tivas más elevadas son las que hacen referencia a: «Conocer y aplicar los fundamentos biológicos, fisiológicos, de maduración evolutiva y el desarrollo psicomotor», «Conocer los elementos y fundamentos de la expresión corporal y la comunicación no verbal y su valor formativo y cultural», «Diseñar un plan de hábitos de vida saludable (higiene y alimentación) y de práctica de actividad física regular» $\mathrm{y}$ «Saber utilizar diferentes instrumentos de evaluación en el área de Educación Física». Se trata de resultados nuevos, lógicos y esperables, dado el contenido de aprendizaje de la asignatura. Pueden encontrarse resultados similares en algunos estudios recientes (Hortigüela et al., 2015; Hortigüela, Abella, Delgado \& Austin, 2016), que encuentran que el alumnado suele tener una valoración positiva y elevada tras la aplicación de sistemas de EFyC, tanto en la percepción de competencias docentes adquirida, como en su percepción de aprendizaje.

\section{Conclusiones}

En conclusión, las principales ventajas encontradas tras la utilización de un sistema de EFyC en una asignatura de FIPEF de una universidad pública chilena son las siguientes: (a) El alumnado realiza una positiva valoración al sistema de EFyC que se implementó durante la asignatura; y (b) El sistema de EFyC parece influir positivamente en la percepción de mejora en la adquisición de competencias «transversales»; «docentes generales»y «docentes específicas de educación física».

Se comprueba que algunas de las características de este tipo de sistemas de evaluación son vividas como inconvenientes por algunos estudiantes, como: «la asistencia continua»; «el trabajo que se puede acumular»; «la desproporción trabajo/créditos». En cambio, para la mayoría del alumnado los inconvenientes reales son escasos y este tipo de sistemas de evaluación se presenta como idóneo en los contextos educativos actuales. Por otra parte, la percepción de desproporción entre trabajo/créditos y carga acumulada parece estar más relacionada con la comparación con sistemas de evaluación tradicional que con las horas reales que el alumnado debe dedicar según los créditos oficiales de cada asignatura.

Las limitaciones del estudio se centran fundamentalmente en lo limitado de la muestra, pero también en la imposibilidad de contrastar los resultados con alumnado que no hubiera tenido otro sistema de evaluación diferente de la EFyC, especialmente con modelos más tradicionales, basados en la impartición de clases magistrales y un examen final.

El estudio puede ser de interés para el profesorado de FIPEF en las universidades chilenas, que imparten su docencia actualmente bajo un modelo competencial, dado que analiza un sistema de evaluación que se presenta como adecuado para la percepción de adquisición de competencias y es altamente valorado por el alumnado. Lógicamente, el sistema de evaluación también puede ser trasferido a universidades de otros países latinoamericanos, por los buenos resultados encontrados en las universidades españolas y chilenas.

En prospectiva futura sería de gran ayuda experimentar sistemas de EFyC en otras asignaturas de FIPEF de la misma 
universidad pública en la que se llevó a cabo el estudio, así como estudiar más experiencias de manera longitudinal en los próximos años. Otra línea de trabajo, compatible con la primera, sería aplicar este tipo de estudios en otras universidades chilenas y analizar los resultados.

\section{Agradecimientos}

El presente artículo es parte del proyecto perteneciente al Programa FONDECYT Iniciación 2019 ํ 11190247, de la Agencia Nacional de Investigación y Desarrollo (ANID).

Titulado: «Hacia la implementación de evaluación formativa y compartida en formación inicial del profesorado de educación física: análisis de sus efectos en titulaciones bajo estructuras curriculares por competencias».

\section{Referencias}

Angelini, L. (2016). Estudio sobre la evaluación formativa y compartida en la formación docente en inglés. Actualidades Investigativas en Educación, 16(1), 1-21. http:// www.scielo.sa.cr/pdf/aie/v16n1/1409-4703-aie-16-0100282.pdf

Atienza, R., Valencia-Peris, A., Martos-García, D., López-Pastor, V. \& Devís-Devís, J. (2016). La percepción del alumnado universitario de educación física sobre la evaluación formativa ventajas, dificultades y satisfacción. Movimento, 22(4), 10331048. Recuperado de https://www.redalyc.org/pdf/1153/ 115349439002.pdf

Barba, J. (2009). Redefiniendo la autoridad en el aula: Posibilidades para una educación democrática. Retos: nuevas tendencias en educación física, deporte y recreación, 15, 41-44. https:// recyt.fecyt.es/index.php/retos/article/view/34997

Barrientos, H., López-Pastor, V., \& Pérez-Brunicardi, D. (2019). ¿Por qué hago evaluación formativa y compartida y/o evaluación para el aprendizaje en EF? La influencia de la formación inicial y permanente del profesorado. Retos: nuevas tendencias en educación física, deporte y recreación, (36), 37-43. Recuperado de https://recyt.fecyt.es/index.php/retos/article/ view/66478

Biggs, J. (2005). Calidad del aprendizaje universitario, Madrid, Narcea.

Boud, D. \& Associates (2010). Assessment 2020: Seven Propositions for Assess-ment Reform in Higher Education. Sydney, Australia Learning and Teaching Council. Recuperado de https://www.uts.edu.au/sites/default/files/Assessment2020 propositions_final.pdf

Burnier, S. (2001). Pedagogia das competencias: Conteuìdos e meitodos. Boletim Teìcnico do Senac, (27). Recuperado de http:/ /www.senac.br/informativo/BTS/27 3/boltec272e.htm

Cabrera, N. \& Mayordomo, R. (2016). El feedback formativo en la universidad. Experiencias con el uso de la tecnología, Barcelona: Colección Transmedia XX.

Calatayud-Salom, M. (2019) Una oportunidad para avanzar hacia la evaluación auténtica en Educación Física. (An opportunity to advance to the authentic evaluation in Physical Education). Retos:nuevas tendencias en educación física, deporte y recreación, 36(36), 259-265. Recuperado de https://recyt.fecyt.es/ index.php/retos/article/view/67540

Canales-Núñez, P., Aravena, O., Carcamo-Oyarzún, J., Lorca, J., \& Martinez-Salazar, C. (2018). Prácticas pedagógicas que favorecen u obstaculizan la inclusión educativa en el aula de educación física desde la perspectiva del alumnado y profesorado. Retos: nuevas tendencias en educación física, deporte y recreación,(34), 212-217. Recuperado de https://recyt.fecyt.es/ index.php/retos/article/view/59620

Castejón-Oliva, F., Santos-Pastor, M. \& Palacios, A. (2015). Cuestionario sobre metodología y evaluación en formación inicial en educación física. Revista Internacional de Medicina y Ciencias de la Actividad Física y del Deporte, 15(58), 245-267. http:// cdeporte.rediris.es/revista/revista58/artescala566.htm

Castejón, F., López-Pastor, V., Julián, J. \& Zaragoza, J. (2011). Evaluación formativa y rendimiento académico en la formación inicial del profesorado de educación física. Revista Internacional de Medicina y Ciencias de la Actividad Física y del Deporte, 11(42), 238-346. Recuperado de http://cdeporte.rediris.es/revista/revista42/artevaluacion163.htm

De Miguel, M. (2005). Cambio de paradigma metodológico en la educación superior: exigencias que conlleva. Cuadernos de Integración Europea, 2, 16-27.

De Winter, J., \& Dodou, D. (2010). Five-Point Likert Items: $t$ test versus Mann-Whitney-Wilcoxon (Addendum added October 2012). Practical Assessment, Research and Evaluation, 15(1), 1-16. Recuperado de https://scholarworks.umass.edu/cgi/ viewcontent.cgi? article $=1237 \&$ context $=$ pare

Declaration, Bolonia (1999). The European Higher Education Area», joint declaration of the European Ministers of Education, convened in Bologna, 19. Recuperado de https:// www.eurashe.eu/library/bologna_1999_bologna-declarationpdf

Delgado, V., Ausin, V., Hortigüela, D. \& Abella-García, V. (2016). Evaluación entre iguales: Una experiencia de evaluación compartida en Educación Superior. EDUCADI, 1(1), 9-24. Recuperado de http://ojs2.uct.cl/index.php/educadi/article/view/943

Díaz-Barriga, Á. (2006). El enfoque de competencias en la educación: ¿Una alternativa o un disfraz de cambio? Perfiles educativos, 28(111), 7-36. Recuperado de http://www.scielo.org.mx/ scielo.php?pid=S0185-26982006000100002

Espinoza, M. (2013). Evaluación de competencias mediante rúbrica. Importancia de las matemáticas en la evaluación de competencias genéricas. Historia y Comunicación Social, 18, 243255. Recuperado de http://revistas.ucm.es/index.php/HICS/ article/viewFile/44240/41802

Fernández-Rivas, M., \& Espada-Mateos, M. (2017). Formación inicial y percepción del profesorado sobre los estilos de enseñanza en Educación Física. Retos: nuevas tendencias en educación física, deporte y recreación, (31), 69-75. Recuperado de https://recyt.fecyt.es/index.php/retos/article/view/49024

Fernández, A. (2010). La evaluación orientada al aprendizaje en un modelo de formación por competencias en la educación universitaria. REDU: Revista de Docencia Universitaria, 8(1), 1135. Recuperado de http://red-u.net/redu/documentos/ vol8_n1_completo.pdf\#page $=11$

Gallardo-Fuentes, F. \& Carter-Thuillier, B. (2016). La evaluación formativa y compartida durante el prácticum en la formación inicial del profesorado: Análisis de un caso en Chile. Retos. Nuevas tendencias en Educación Física Deportes y Recreación, (29), 258-263. Recuperado de https://recyt.fecyt.es/ index.php/retos/article/view/43550

Hamodi, C., López, A. \& López-Pastor, V. (2014). Red de evaluación formativa y compartida en docencia universitaria: creación, consolidación y líneas de trabajo. REVALUE. Revista de Evaluación Educativa, 3(1). Recuperado de http:// ojs.inee.edu.mx/revista/index.php/revalue/article/view/110

Hawes, G. \& Corvalán, O. (2005). Construcción de un perfil profesional. Instituto de Investigación y Desarrollo Educacional. Proyecto Mecesup Tal0101, Talca, Universidad de Talca.

Hortigüela, D., Abella, V., Delgado, V. \& Austin, V. (2016). Influencia del sistema de evaluación empleado en la percepción del alumno sobre su aprendizaje y las competencias docentes. Revista Infancia, Educación y Aprendizaje, 2(1), 20-42. Recuperado de http://portals3.uv.cl/index.php/IEYA/article/view/ 
$582 / 576$

Hortigüela, D., Pérez-Pueyo, Á. \& Abella, V. (2015). ¿De qué manera se implica el alumnado en el aprendizaje? Análisis de su percepción en procesos de evaluación formativa. Revista de Investigación en Educación, 13(1), 88-104. Recuperado de http://reined.webs.uvigo.es/ojs/index.php/reined/article/ viewFile/1002/347

Hortigüela, D., Pérez-Pueyo, Á. \& López-Pastor, V. (2015). Implicación y regulación del trabajo del alumnado en los sistemas de evaluación formativa en educación superior. RELIEVE. Revista Electrónica de Investigación y Evaluación Educativa, 21(19), 1-5. doi:10.7203/relieve.21.1.5171

Julián, J., Zaragoza, J., Castejón-Oliva, F. \& López-Pastor, V. (2010) Carga de trabajo en diferentes asignaturas que experimentan el sistema ECTS. Revista Internacional de Medicina y Ciencias de la Actividad Física y el Deporte, 10(38), 218-233. Recuperado de Http://cdeporte.rediris.es/revista/revista38/ artcarga151.htm

Lasnier, F. (2000). Réussir la formation par compétences, Montréal, Guérin.

López-Pastor, V. (2008). Desarrollando sistemas de evaluación formativa y compartida en la docencia universitaria. Análisis de resultados de su puesta en práctica en la formación inicial del profesorado. European Journal of Teacher Education, 31(3), 293-311. doi: http://dx.doi.org/10.1080/ 02619760802208452

López-Pastor, V. (2011). El papel de la evaluación formativa en la evaluación por competencias: aportaciones de la red de evaluación formativa y compartida en docencia universitaria. REDU. Revista de Docencia Universitaria, 9(1), 159. doi: http:/ dx.doi.org/10.4995/redu.2011.6185

López-Pastor, V. (coord.) (2009). Evaluación formativa y compartida en Educación Superior, Madrid: Narcea.

López-Pastor, V. \& Pérez-Pueyo, Á. (coord.) (2017). Evaluación formativa y compartida en educación: experiencias de éxito en todas las etapas educativas, León: Universidad de León. ebook, Recuperado de https://buleria.unileon.es/handle/10612/ 5999

López-Pastor, V., Pérez, Á., Barba, J. \& Lorente, E. (2016). Percepción del alumnado sobre la utilización de una escala graduada para la autoevaluación y coevaluación de trabajos escritos en la formación inicial del profesorado de educación física (FIPEF). Cultura, ciencia y deporte, 11(31), 37-50. Recuperado de http://www.redalyc.org/pdf/1630/163044427005.pdf

López-Pastor, V., Pintor, P., Muros, B. \& Webb, G. (2013). Formative assessment strategies and their effect on student performance and on student and tutor workload: the results of research projects undertaken in preparation for greater convergence of universities in Spain within the European Higher Education Area (EHEA). Journal of Further and Higher Education, 37(2), 163-180. doi:http://dx.doi.org/10.1080/ 0309877X.2011.644780

López, C., Benedito, V., \& León, M. (2016). El enfoque de competencias en la formación universitaria y su impacto en la evaluación: la perspectiva de un grupo de profesionales expertos en Pedagogía. Formación universitaria, 9(4), 11-22. doi: 10.4067/ S0718-50062016000400003

Martín, P. (2007). Evaluación formativa y su repercusión en el clima del aula. Revista de Investigación educativa, 25(2), 389402. Recuperado de https://revistas.um.es/rie/article/view/96851

Martínez-Mínguez, L. (2016). Proyectos de Aprendizaje Tutorados y autoevaluación de competencias profesionales en la formación inicial del profesorado. Retos: nuevas tendencias en educación física, deporte y recreación, (29), 242-250. Recuperado de https://recyt.fecyt.es/index.php/retos/article/view/42719/ 25484

Martínez, L., Santos-Pastor, M., \& Castejón-Oliva, F. (2017). Percepciones de alumnado y profesorado en Educación Supe- rior sobre la evaluación en formación inicial en educación física. Retos. Nuevas Tendencias en Educación Física, Deporte y Recreación, (32), 76-81. Recuperado de https://recyt.fecyt.es/ index.php/retos/article/view/52918

Perrenoud, P. (2002). Construir competencias desde la escuela. $2^{a}$. Edición, Santiago de Chile: Dolmen Ediciones.

Proyecto Tunning (2003). Tunning Educational Structure in Europe. Informe final, Bilbao, Universidad de Deusto.

Rodríguez, I., Del Valle, S., \& De la Vega, R. (2018). Revisión nacional e internacional de las competencias profesionales de los docentes de Educación Física. Retos: nuevas tendencias en educación física, deporte y recreación, (34), 383-388. Recuperado de https://recyt.fecyt.es/index.php/retos/article/view/ 58609

Romero-Martín, R., Castejón-Oliva, F. \& López-Pastor, V. (2015). Divergencias del alumnado y del profesorado universitario sobre las dificultades para aplicar la evaluación formativa. $R E$ LIEVE-Revista Electrónica de Investigación y Evaluación Educativa, 21(1), 1-16. doi:10.7203/relieve.21.1.5169

Romero-Martín, R., Fraile-Aranda, A., López-Pastor, V. \& Castejón-Oliva, F. (2014). Relación entre sistemas de evaluación formativa, rendimiento académico y carga de trabajo del profesor y del alumno en la docencia universitaria. Infancia y Aprendizaje, 37(2), 310-341. doi:http://dx.doi.org/10.1080/ 02103702.2014 .918818

Salcines-Talledo, I., González-Fernández, N., Ramírez-García, A. \& Martínez-Mínguez, L. (2018). Validación de la escala de autopercepción de competencias transversales y profesionales de estudiantes de educación superior. Profesorado, Revista de Currículum y Formación del Profesorado, 22(3), 31-51. Recuperado de https://digibug.ugr.es/handle/10481/53207

Santos-Pastor, M., Martínez-Muñoz, L. \& López-Pastor, V. (coord.) (2009). La innovación docente en el EEES: experiencias de evaluación formativa y compartida en la formación inicial del profesorado, Almería: Editorial Universidad de Almería.

Senye-Mir, A., Arumí-Prat, J., Pla-Campas, G. \& Ramírez, E. (2016). Effects of Formative Assessment on the Learning-tolearn Skills of Teacher Training Students. Procedia-Social and Behavioral Sciences, 228, 196-201. doi: https://doi.org/10.1016/ j.sbspro.2016.07.029

Silva, I. \& López-Pastor, V. (2015). ¿Cómo vive el alumnado la evaluación en formación inicial del profesorado?. @tic. Revista d'innovació educativa, (14), 1-11. doi:10.7203/attic.14.4171

Tejada, J. (2009). Competencias docentes. Profesorado, Revista de Currículum y Formación del Profesorado, 13(2), 1-15. Recuperado de https://www.ugr.es/ recfpro/rev132COL2.pdf

Tobón, S., Rial, A., Carretero, M. \& García, J. (2006). Competencias, calidad y educación superior, Bogotá: Cooperativa Editorial Magisterio.

Ureña-Ortín, N. \& Ruiz-Lara, E. (2012). Experiencia de evaluación formativa y compartida en el Máster Universitario en Formación del Profesorado de Educación Secundaria. Psychology, Society \& Education, 4(1), 29-44.

Vallés, C., Ureña, N. \& Ruiz, E. (2011). La Evaluación Formativa en Docencia Universitaria. Resultados globales de 41 estudios de caso». RED-U. Revista de Docencia Universitaria, 9(1), 135-158. Recuperado de http://red-u.net/redu/files/journals/1/ articles/197/public/197-203-2-PB.pdf

Yániz, C. \& Villardón, L. (2006). Planificar desde competencias para promover el aprendizaje, Bilbao: Universidad de Deusto.

Zabalza, M. (2003). Competencias docentes del profesorado universitario. Calidad y desarrollo profesional, Madrid: Narcea.

Zaragoza, J., Luis-Pascual, J. \& Manrique, J. (2009). Experiencias de innovación en docencia universitaria: resultados de la aplicación de sistemas de evaluación formativa. RED-U. Revista de Docencia Universitaria, 7(4), 1-33. Recuperado de https:// revistas.um.es/redu/article/view/92561 